Annals of Pure and Applied Mathematics

Vol. 16, No. 1, 2018, 127-131

ISSN: 2279-087X (P), 2279-0888(online)

Published on 5 January 2018

www.researchmathsci.org

DOI: http://dx.doi.org/10.22457/apam.v16n1a14

Annals of

Pure and Applied

Mathematics

\title{
Left Generalized Derivations on Prime $\Gamma$-Rings
}

\author{
C. Jaya Subba Reddy ${ }^{1}$, K. Nagesh ${ }^{2}$ and A. Sivakameshwara Kumar ${ }^{3}$ \\ ${ }^{1}$ Department of Mathematics, Sri Venkateswara University \\ Tirupati-517502, India. E-mail: cjsreddysvu@ gmail.com \\ ${ }^{2}$ Department of Mathematics, Rayalaseema University \\ Kurnool, Andhrapradesh, India. E-mail: nagesh.kunda100@gmail.com \\ ${ }^{3}$ Department of Mathematics, Rayalaseema University \\ Kurnool, Andhrapradesh, India. E-mail: kamesh1069@ gmail.com
}

Received 6 December 2017; accepted 26 December 2017

Abstract. Let $M$ be a prime $\Gamma$-ring with 2-torsion free, $I$ a nonzero ideal of $\mathrm{M}$ and $f: M \rightarrow M$ a left generalized derivation of $M$, with associated nonzero derivation $\mathrm{d}$ on $M$. If $f(x) \in Z(M)$ for all $x \in I$, then $M$ is a commutative $\Gamma$-ring.

Keywords: Gamma ring, prime gamma ring, derivation, generalized derivation, left generalized derivation, commutators.

\section{AMS Mathematics Subject Classification (2010): 06F25}

\section{Introduction}

The notion of $\Gamma$-ring was first introduction by Nobusawa [9] and also shown that $\Gamma$-ring, more general than rings. Barnes [1] slightly weakened the conditions in the definitions of a $\Gamma$-rings in the sense of Nobusawa. After the study of $\Gamma$-rings by Nobusawa [9] and Barnes [1], many researchers have a done lot of work and have obtained some generalizations of the corresponding results in ring theory [6][8]. Barnes [1] and kyuno [8] studied the structure of $\Gamma$-ring and obtained various generalizations of the corresponding results of ring theory. Hvala [4] introduced the concept of Generalized derivations in rings. Dey, Paul and Rakhimov [3] discussed some properties of Generalized derivations in semiprime gamma rings Bresar [2] studied on the distance of the composition of two derivations to the generalized derivations. Jaya Subba Reddy. et al. [5] studied centralizing and commutating left generalized derivation on prime ring is commutative. Jaya Subba Reddy et al. [12] studied some results of symmetric reverse bi-derivations on prime rings, Ozturk et al. [10] studied on derivations of prime gamma rings. Khan et al. [6,7] studied on derivations and generalized derivations on prime $\Gamma$ rings is a commutative. In this paper we extended some results on left generalized derivations on prime $\Gamma$-ring is a commutative.

\section{Preliminaries}

If $M$ and $\Gamma$ are additive abelian groups and there exists a mapping $M \times \Gamma \times M \rightarrow M$ which satisfies the following conditions:

For all $a, b \in M$ and $\alpha, \beta \in \Gamma$, 


\section{Jaya Subba Reddy, K. Nagesh and A. Sivakameshwara Kumar}

(i) $(a, \beta, b)$, denoted by $a \beta b$, is an element of $M$

(ii) $(a+b) \beta c=a \beta c+b \beta c, a(\alpha+\beta) b=a \alpha b+a \beta b, a \beta(b+c)=a \beta b+a \beta c$

(iii) $(a \alpha b) \beta c=a \alpha(b \beta c)$

then $M$ is called a $\Gamma$-ring [1]. It is known that from (i), (iii) the following follows:

$0 \beta b=a 0 b=a \beta 0=0$

for all $a$ and $b$ in $M$ and all $\beta$ in $\Gamma$ [1].

Every ring is a $\Gamma$-ring with $M=\Gamma$. However a $\Gamma$-ring need not be a ring. Let $M$ be a $\Gamma$ ring, then $M$ is called a prime $\Gamma$-ring, if $a \Gamma M \Gamma b=0$ implies $a=0$ or $b=0$, for all $a, b \in M$ and $M$ is called a semiprime $\Gamma$-ring, if $a \Gamma M \Gamma a=0$ implies $a=0$, for all $a \in$ $M$. Every prime $\Gamma$-ring is obviously semiprime. If $M$ is a $\Gamma$-ring, then $M$ is said to be 2torsion free if $2 x=0$ implies $x=0$, for all $x \in M$. An additive subgroup $I$ of $M$ is called a left (right) ideal of $M$ if $M \Gamma I \subseteq I$ (IГM $\subseteq \mathrm{I}$ ). If $I$ is both left and right ideal of $M$, then we say $I$ is an ideal of $M$. Moreover, the set $Z(M)=\{x \in M: x \beta y=y \beta x \quad \forall \beta \in$ $\Gamma, \mathrm{y} \in \mathrm{M}\}$ is called the centre of the $\Gamma$-ring $M$. We shall write $[x, y]_{\beta}=x \beta y-y \beta x$, for all $x, y \in M$ and $\beta \in \Gamma$. We shall make use of the basic commutator identities: $[x \beta y, z]_{\alpha}=[x, z]_{\alpha} \beta y+x \beta[y, z]_{\alpha}$ and $[x, y \beta z]_{\alpha}=[x, y]_{\alpha} \beta z+y \beta[x, z]_{\alpha}$, for all $x, y, z \in M$ and $\alpha, \beta \in \Gamma$. If $\Gamma$-ring satisfies the assumption (B) $a \alpha b \beta c=a \beta b \alpha c$, for all $a, b, c \in M$ and $\alpha, \beta \in \Gamma$. Let $M$ be a $\Gamma$-ring. An additive mapping $d: M \rightarrow M$ is called a derivation on $M$ if $d(x \gamma y)=d(x) \gamma y+x \gamma d(y)$ holds for all $x, y \in M$ and $\gamma \in \Gamma$. An additive mapping $f: M \rightarrow M$ is called a generalized derivation if there exists a derivation $d: M \rightarrow M$ such that $f(x \gamma y)=f(x) \gamma y+x \gamma d(y)$ holds for all $x, y \in M$ and $\gamma \in \Gamma$. An additive mapping $f: M \rightarrow M$ is called a left generalized derivation if there exists a derivation $d: M \rightarrow M$ such that $f(x \gamma y)=x \gamma f(y)+d(x) \gamma y$ holds for all $x, y \in M$ and $\gamma \in \Gamma$. A derivation of the form $x \rightarrow a \alpha x+x \alpha b$ where $a, b$ are fixed elements of $M$ and $\alpha \in \Gamma$ is called generalized inner derivation. An additive mapping $T: M \rightarrow M$ is called a left (right) centralizer if $T(x \alpha y)=T(x) \alpha y(T(x \alpha y)=x \alpha T(y))$ for all $x, y \in M$ and $\alpha \in \Gamma$.

Lemma 2.1. Let $M$ be a prime $\Gamma$-ring with 2-torsion free and $I$ a nonzero ideal of $M$. Let $f: M \rightarrow M$ be a left generalized derivation of $M$, associated with derivation d. If $f(y)=$ 0 , for all $y \in I$, then $f=0$.

Proof: For all $x, y \in I$ and $\beta \in \Gamma, f(x \beta y)=0$. That is, $x \beta f(y)+d(x) \beta y=0$, which implies $d(x) \beta y=0$. Let $z \in M, \alpha \in \Gamma$. The last relation along with (A) gives, $d(x) \beta z \alpha y=0$. Since $M$ is prime $\Gamma$-ring and $I$ is a nonzero ideal, so $d(x)=0$, for all $x \in I$. Hence, by hypothesis, $f(x \beta r)=0$, for all $x \in I$, and $\beta \in \Gamma$, and $r \in M$. That is, $x \beta f(r)+d(x) \beta r=0$, which gives $x \beta f(r)=0$. Let $w \in M, \gamma \in \Gamma$. The last relation along with (A), implies $x \gamma w \beta f(r)=0$. Since $I$ is nonzero and primeness of $M$, gives $f=0$.

Lemma 2.2. Let $I$ be a nonzero ideal of a prime $\Gamma$-ring $M, a \in M$ and $f \neq 0$ is a left generalized derivation of $M$, with associated nonzero derivation $d$, then

(i) If $f(y) \beta a=0$ for all $y \in I$ and $\beta \in \Gamma$, then $a=0$,

(ii)If $a \beta f(y)=0$ for all $y \in I$ and $\beta \in \Gamma$, then $a=0$.

Proof: 


\section{Left Generalized Derivations on Prime $\Gamma$-Rings}

(i) For any $y \in I, r \in M$ and $\alpha, \beta \in \Gamma, f(r \alpha y) \beta a=0$. That is, $r \alpha f(y) \beta a+d(r) \alpha y \beta a=0$ Which implies, $d(r) \alpha y \beta a=0$. Since $I$ is a nonzero ideal of $M$ and $d \neq 0$, we get $a=0$.

(ii) Proof is similar to (i).

\section{Main results}

Theorem 3.1. Let $M$ be a prime $\Gamma$-ring with 2-torsion free and $I$ a nonzero ideal of $M$. Let $f: M \rightarrow M$ be a left generalized derivation of $M$, with associated nonzero derivation d on $M$. If $f(x) \in Z(M)$ for all $x \in I$, then $M$ is a commutative $\Gamma$-ring.

Proof: Using hypothesis, we have $[f(x \beta y), x]_{\alpha}=0$, for all $x, y \in I, \alpha, \beta \in \Gamma$, which gives $[x \beta f(y)+d(x) \beta y, x]_{\alpha}=0$

$$
x \beta[f(y), x]_{\alpha}+[x, x]_{\alpha} \beta f(y)+d(x) \beta[y, x]_{\alpha}+[d(x), x]_{\alpha} \beta y=0
$$

Using hypothesis, we get

$$
\begin{aligned}
& d(x) \beta[y, x]_{\alpha}+[d(x), x]_{\alpha} \beta y=0 \\
& d(x) \beta y \alpha x-d(x) \beta x \alpha y+d(x) \alpha x \beta y-x \alpha d(x) \beta y=0
\end{aligned}
$$

Using (B), from the last equation we get

$$
\begin{aligned}
& d(x) \beta y \alpha x-d(x) \alpha x \beta y+d(x) \alpha x \beta y-x \alpha d(x) \beta y=0 \\
& d(x) \beta y \alpha x-x \alpha d(x) \beta y=0, \text { for all }, y \in I, \alpha, \beta \in \Gamma .
\end{aligned}
$$

Let $z \in I$. Replacing $y$ by $z \beta y$ in equation (1), we get

$d(x) \beta z \beta y \alpha x-x \alpha d(x) \beta z \beta y=0$

Which along with equation (1) and (B) gives ,

$d(x) \beta z \beta y \alpha x-d(x) \beta z \beta x \alpha y=0$

$d(x) \beta z \beta[y, x]_{\alpha}=0$, for all $x, y \in I$ and $\alpha, \beta \in \Gamma$.

Since $I$ is a nonzero ideal of $M$ and $d \neq 0$, therefore $M$ is a commutative $\Gamma$ - ring.

Theorem 3.2. Let $M$ be a prime $\Gamma$-ring with 2-torsion free and $I$ a nonzero ideal of $M$. Let $f: M \rightarrow M$ be a generalized derivation and left generalized derivation of $M$, with associated derivation $\mathrm{d}$ on $M$. If $a \in M$ and $[f(x), a]_{\alpha}=0$, for all $x \in I, \alpha \in \Gamma$, then either $a \in Z(M)$ or $d(a)=0$.

Proof: Using hypothesis, we have

$[f(x \beta y), a]_{\alpha}=0$, for any $x \in M, y \in I$ and $\alpha, \beta \in \Gamma$.

This gives $[x \beta d(y)+f(x) \beta y, a]_{\alpha}=0$

$[x \beta d(y), a]_{\alpha}+[f(x) \beta y, a]_{\alpha}=0$

The last equation gives

$$
x \beta[d(y), a]_{\alpha}+[x, a]_{\alpha} \beta d(y)+f(x) \beta[y, a]_{\alpha}+[f(x), a]_{\alpha} \beta y=0
$$

Using hypothesis, from the last equation we get

$$
\begin{aligned}
& x \beta[d(y), a]_{\alpha}+[x, a]_{\alpha} \beta d(y)+f(x) \beta[y, a]_{\alpha}=0 \\
& x \beta d(y) \alpha a-x \beta a \alpha d(y)+x \alpha a \beta d(y)-a \alpha x \beta d(y)+f(x) \beta y \alpha a-f(x) \beta a \alpha y=0 \\
& \text { Using (B), from the last equation we get } \\
& x \beta d(y) \alpha a-x \alpha a \beta d(y)+x \alpha a \beta d(y)-a \alpha x \beta d(y)+f(x) \beta y \alpha a-f(x) \beta a \alpha y=0 \\
& x \beta d(y) \alpha a-a \alpha x \beta d(y)+f(x) \beta y \alpha a-f(x) \beta a \alpha y=0 \\
& \text { Let } z \in M \text {. Replacing } x \text { by } z \gamma x \text { in equation }(2), \text { we get } \\
& z \gamma x \beta d(y) \alpha a-a \alpha z \gamma x \beta d(y)+f(z \gamma x) \beta y \alpha a-f(z \gamma x) \beta a \alpha y=0 \\
& z \gamma x \beta d(y) \alpha a-a \alpha z \gamma x \beta d(y)+z \gamma(f(x) \beta y \alpha a-f(x) \beta a \alpha y)+d(z) \gamma x \beta(y \alpha a-a \alpha y) \\
& =0
\end{aligned}
$$

Using equation (2), from the last equation we get 


\section{Jaya Subba Reddy, K. Nagesh and A. Sivakameshwara Kumar}

$$
\begin{aligned}
& z \gamma x \beta d(y) \alpha a-\operatorname{a\alpha z} \gamma x \beta d(y)+z \gamma(a \alpha x \beta d(y)-x \beta d(y) \alpha a)+d(z) \gamma x \beta[y, a]_{\alpha}=0 \\
& z \gamma x \beta d(y) \alpha a-\operatorname{a\alpha z\gamma x} \beta d(y)+z_{\gamma} \operatorname{a\alpha x} \beta d(y)-z \gamma x \beta d(y) \alpha a+d(z) \gamma x \beta[y, a]_{\alpha}=0 \\
& \text { Using (B), from the last equation we get }
\end{aligned}
$$

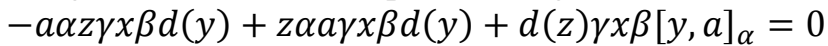

$$
\begin{aligned}
& {[z, a]_{\alpha} \gamma x \beta d(y)+d(z) \gamma x \beta[y, a]_{\alpha}=0}
\end{aligned}
$$

Corollary 3.2.1. Let $M$ be a prime $\Gamma$-ring with 2-torsion free and $I$ a nonzero ideal of $M$. Let $f: M \rightarrow M$ be a left generalized derivation of $M$, with associated derivation d on $M$. If $[f(x), f(y)]_{\beta}=0$, for all $x, y \in I, \beta \in \Gamma$, then $M$ is a commutative $\Gamma$-ring.

Proof: Using Theorem 3.2, we have $f(I) \subset Z(M)$, we get the corollary 3.2.1 proof.

Theorem 3.3. Let $M$ be a prime $\Gamma$-ring with 2-torsion free and $I$ a nonzero ideal of $\quad M$. Let $f: M \rightarrow M$ be a left generalized derivation of $M$, with associated derivation d on $M$. If $f(x \beta y)=f(x) \beta f(y)$, for all $x, y \in I, \beta \in \Gamma$, then $d=0$.

Proof: $f(x \beta y)=x \beta f(y)+d(x) \beta y$, for any $x, y \in I, \beta \in \Gamma$.

$f(x) \beta f(y)=x \beta f(y)+d(x) \beta y$

Let $w \in I, \gamma \in \Gamma$.Then replacing $y$ by $w \gamma y$ in equation (3), we get

$f(x) \beta f(w \gamma y)=x \beta f(w \gamma y)+d(x) \beta w \gamma y$

$d(x) \beta w \gamma(f(y)-y)=0$, for all $x, y \in I$, and $\gamma, \beta \in \Gamma$.

Since $I$ is a nonzero ideal of the prime $\Gamma$-ring $M$, therefore either $f(y)-y=0$ for all $y \in I$ or $d(x)=0$ for all $x \in I$. If $f(y)-y=0$, then $f(y)=y$ for all $y \in I$. Replacing $y$ by $y \beta x$ in the last equation, we get $f(y \beta x)=y \beta x$, which implies $y \beta f(x)+$ $d(y) \beta x=y \beta x$, which gives $y \beta x+d(y) \beta x=y \beta x$. That is $d(y) \beta x=0$, for all $x, y \in I$, $\beta \in \Gamma$. Thus $d(y)=0$ for all $y \in I$ for both cases. So $d=0$.

Theorem 3.4. Let $M$ be a prime $\Gamma$-ring with 2-torsion free and $I$ a nonzero ideal of $M$. Let $f: M \rightarrow M$ be a left generalized derivation of $M$, with associated derivation d on $M$. If $f(x \beta y)=f(y) \beta f(x)$, for all $x, y \in I, \beta \in \Gamma$, then $d=0$.

Proof: $f(x \beta y)=x \beta f(y)+d(x) \beta y$, for all $x, y \in I, \beta \in \Gamma$.

$f(y) \beta f(x)=x \beta f(y)+d(x) \beta y$

Let $x \in I, \gamma \in \Gamma$. Replacing $y$ by $x \gamma y$ in equation (4), we get

$f(x \gamma y) \beta f(x)=x \beta f(x \gamma y)+d(x) \beta x \gamma y$

$x \gamma f(y) \beta f(x)+d(x) \gamma y \beta f(x)=x \beta f(y) \gamma f(x)+d(x) \beta x \gamma y$

Using (B), from the last equation we get

$x \beta f(y) \gamma f(x)+d(x) \gamma y \beta f(x)=x \beta f(y) \gamma f(x)+d(x) \beta x \gamma y$

$d(x) \gamma y \beta f(x)=d(x) \beta x \gamma y$

Let $w \in I, \alpha \in \Gamma$. Then replacing $y$ by $y \alpha w$, we get

$d(x) \gamma y \alpha w \beta f(x)=d(x) \beta x \gamma y \alpha w$

Using equation (5) in above equation, we get

$d(x) \gamma y \alpha w \beta f(x)=d(x) \gamma y \beta f(x) \alpha w$

Using (B), from the last equation we get 
Left Generalized Derivations on Prime $\Gamma$-Rings

$$
\begin{aligned}
& d(x) \gamma y \alpha f(x) \beta w-d(x) \gamma y \alpha w \beta f(x)=0 \\
& d(x) \gamma y \alpha[f(x), w]_{\beta}=0
\end{aligned}
$$

Since $I$ is a nonzero ideal of the prime $\Gamma$-ring $M$. Therefore either $d(x)=0$ for all $x \in I$ or $[f(x), w]_{\beta}=0$ for all $x, w \in I$ and $\beta \in \Gamma$. Let $A=\{x \in I: d(x)=0\}$ and $B=$ $\left\{x \in I:[f(x), w]_{\beta}=0, \forall w \in I\right\}$. Obviously $A$ and $B$ are additive subgroups of $I$. Moreover $I$ is the set theoretic union of $A$ and $B$. But a group cannot be set theoretic union of two proper sub groups. Hence either $A=I$ or $B=I$. If $A=I$, we have $d(R)=0$, which completes the proof. If $B=I$, then $0=[f(x), w]_{\beta}=w \alpha[f(x), r]_{\beta}$ for all $x, w \in I, \quad r \in M$ and $\alpha, \beta \in \Gamma$. Thus, we obtain $f(I) \subset Z(M)$, Using Theorem 3.2 , we get $d=0$.

Acknowledgements. We are thankful to the reviewers for their comments to improve the presentation of the paper.

\section{REFERENCES}

1. W.E.Barnes, On the $\Gamma$-rings of Nobusawa, Pacific J. Math., 18 (3) (1966) 411-422.

2. M.Bresar, On the distance of the composition of two derivations to the generalized Derivations, Glasgow Math. J., 33 (1) (1991) 89-93.

3. K.K.Dey, A.C.Paul and I.S.Rakhimov, Generalized derivations in semiprime gamma rings, IJMMS, 2012.

4. B.Hvala, Generalized derivations in rings, Comm. Algebra, 26 (4) (1998) 1147-1166.

5. C.Jaya Subba Reddy, S.Mallikarjuna Rao and V.Vijaya Kumar, Centralizing and commuting left generalized derivations on prime rings, Bulletin of Mathematical Science and Applications, 11 (2015) 1-3.

6. A.R.Khan, M.Anwar Chaudhry and Imran Javaid, Generalized derivations on prime T-rings, World Applied Sciences Journal, 23 (12) (2013) 59-64.

7. A.R.Khan, I.Javaid and M.Anwar Chaudhry: Derivations on semiprime $\Gamma$-rings, Utilitas Mathematica, 90 (2013) 171-185.

8. S.Kyuno, On prime gamma rings, Pacific J. Math., 75 (1) (1978) 185-190.

9. N.Nobusawa, On the generalization of the ring theory, Osaka J. Math., 1 (1964) 8189.

10. M.A.Ozturk, Y.B.Jun and K.H.Kim, On derivations of prime gamma rings. Turk. J. Math., 26 (2002) 317-327.

11. C.Yilmaz, and M.A.Ozturk, On Jordan generalized derivations in gamma rings, Hacet. J. Math. Stat.,33 (2004) 11-14.

12. C. Jaya Subba Reddy, A. Sivakameshwara Kumar and B. Ramoorthy Reddy, Results of Symmetric Reverse bi-derivations on Prime Rings, Ann. Pure and Applied Math,16(1)(2018) 1-6.

13. A.K.Kadhim,H.Sulaiman and A.R.H.Majeed, Gamma * derivation pair and jordan gamma*- derivation pair on gamma-ring $M$ with involution, Ann. Pure and Applied Math, 10(2) (2015) 169-177. 